\title{
eINTERASIA Project: IT Transfer Concept for Adaptation and Dissemination of Innovative European Research Results in Central Asian Countries
}

\author{
Leonids Novickis ${ }^{1}$, Antanas Mitasiunas ${ }^{2}$, \\ ${ }^{1}$ Riga Technical University, Latvia, ${ }^{2}$ MitSoft Ltd., Vilnius University, Lithuania
}

\begin{abstract}
The paper presents an overview of the eINTERASIA international project aimed at supporting international cooperation with Central Asian countries by transfer, adaptation and dissemination of European research results in the area of Information Technology and Applied Software.

Information Technology Transfer Concept (TTC) based on InnoSPICE approach and Capability Maturity Model has been developed.

The concept of showroom as part of the implementation of TTC has been introduced in the selected Central Asian countries. Technological platforms for demonstrators of Virtual RealityBased scenarios from Fraunhofer IFF were adapted and installed in the region of Central Asia.

Several EU research projects are selected for adaptation and validation in Central Asian countries. Special attention is devoted to demonstrate transfer and adaptation processes in the area of web-based transport logistics.
\end{abstract}

Keywords - International cooperation, information technology transfer, web-based transport logistics.

\section{INTRODUCTION}

The eINTERASIA project runs from March $1^{\text {st }}, 2013$ to August $31^{\text {st }}, 2015$ under the $7^{\text {th }}$ Framework Program of the European Commission.

The major purpose of eINTERASIA is to support international cooperation with Central Asian countries by creating a Technology Transfer Concept for adaptation, piloting, diffusion and local exploitation of the EU research results. The application of TTC will be demonstrated in the field of eLogistics.

The project consists of five main phases (Fig. 1):

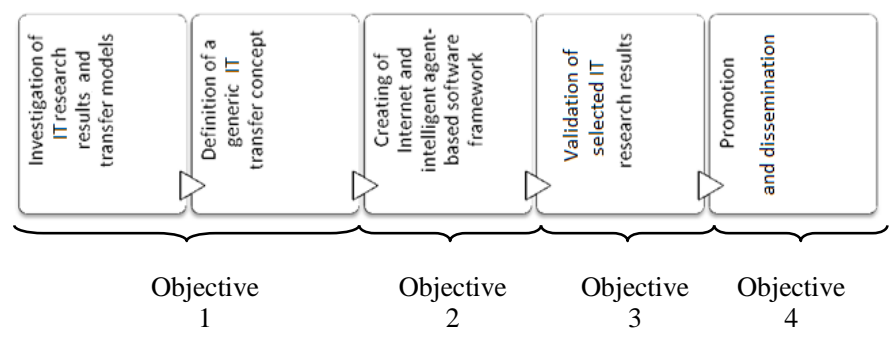

Fig. 1. Three main phases of the project.
Objective 1: Investigating technology transfer models and IT research results; defining the generic IT transfer concept.

The transfer model is based on a Capability Maturity Model, ISO standards and the best current practices in fields such as communication, knowledge and project management and commercialization of research results. It helps corresponding organizations characterize their capability in current transfer practice and establish the transfer strategy of research findings [1].

In the process of investigation several EU research projects were selected for further adaptation and piloting in Central Asian countries:

- InnoSPICE: Knowledge- and Technology Transfer Process Capability Model [2];

- eLogmar: Web-based Portal in Transport and Logistics [3];

- VR: Virtual Reality Platforms and Models [4];

- SIGNA: Document e-Signature System [5].

Objective 2: Creating software frameworks and developing an intelligent multi-agent model to support business processes in the application domain of transport logistics.

The results of investigation of EU projects serve as a platform for creating a multi-agent and Web-based Framework (WBF) to support selected business applications.

General logistics was analyzed, formalized and presented in the form of "essential logistics principles" conducive for business process re-engineering and adaptation to local conditions of the Central Asian countries. This business model was used for creating an intelligent multi-agent based software framework.

eINTERASIA software framework (web portal) includes different electronic resources:

- Web portal www.elogmar.eu (the output of the EU research project eLogmar-M [3]) adapted to the needs of the target region of Central Asia;

- Cargo auction mode based on Java multi-agent model;

- Thematic databases containing information on IT \& Mobile Solutions and Education and Training;

- Information on the selected EU research results.

Objective 3: Validation of selected IT research results. 
Validation process runs from March $1^{\text {st }}$ to June $30^{\text {th }} 2015$. In the course of the validation, on the basis of the data obtained from the potential users of the proposed products/services, the level of fulfilment of the requirements for specific applications is assessed.

The primary medium of the user feedback collection is the questioning. Subsequently, the data gained in the course of the questioning are processed and will be submitted in the form of the Validation Report at the end of the validation process.

Validation is implemented with regard to the following:

a) Technology Transfer Concept (TTC);

b) TTC from end user's point of view;

c) Selected IT solution/services of EU partners (InnoSPICE, eLOGMAR, VR, SIGNA).

Both the EU representatives and Central Asian organizations/individuals that the respective IT solutions were transferred to participate in the validation process.

Final validation results will be presented in the form of the project "Newspaper".

\section{Objective 4: Promotion and dissemination.}

The following activities are implemented:

- Defining and implementing a strategy to capture the project outputs and to present them within the European and Central Asian economies and ICT communities;

- Promoting the transfer of know-how and best practices;

- Collaborating with international research networks and ongoing EU and national projects;

- Promoting the advanced IT solutions at international conferences and exhibitions;

- Generalizing results of investigations in the form of "News Journal" (three volumes);

- Disseminating the research knowledge and project results via mass media.

\section{Consortium}

Nine partners from seven countries are represented in the consortium:

- Riga Technical University (RTU, Latvia);

- University of Bremen (UniB, Germany);

- Fraunhofer IFF Institute for Factory Operation and Automation (IFF, Germany);

- Logitrans Consult Ltd. (LTR, Estonia);

- MitSoft Ltd. (MITSOFT, Lithuania);

- Astana Innovations (AI, Kazakhstan);

- BISAM Central Asia (BISAM, Kazakhstan);

- Uzbek International Forwarders Association (UIFA, Uzbekistan);

- Technological University of Tajikistan (TUT, Tajikistan).

This combination brings together the complementary expertise and skills of partner organizations active in IT, applied research and international cooperation.

\section{SITUATION IN INNOVATION AND TECHNOLOGY TRANSFER MODELS AND INTERNATIONAL COOPERATION ACTIVITIES}

IT transfer concepts are based on general principles of innovation and technology transfer process and models. From the general point of view, innovation can be understood as a process from idea generation to commercialization - bringing the idea or invention to the market as a new product or service through the phases of idea generation, research and development, product/service development, marketing and selling. To turn those inventions into successful innovations, they must be transferred from academia to industry. Six generations of innovation process models have been developed ranging from simple linear models to complex interactive models [6]-[10]: Technology Push, Market Pull, Coupling Model, Interactive Model, Network Model, Open Innovation, CMM.

There are several international cooperation projects funded by the EC dealing with ICT promotion in developing countries and using some of innovation process models:

- IncrEAST is a web-based information platform designed to intensify international cooperation in S\&T and to facilitate the networking of research organizations in the countries of Eastern Europe/Central Asia and the EU. It provides up-to-date information about the political development of research in the targeted countries; however, not all countries are covered by the so-called "Hosting Institutes";

- Ami@work provides a collaborative environment that supports the development of promising initiatives and aims at increasing the impact of the European Research and Innovation Area;

- EuroAfrica-ICT is aimed at cooperation on e-infrastructure between the EU and Africa;

- Several EU projects (DENEMA, TELEINVIVO, TELESOL) dealing with application of telematics solutions for promoting the cooperation between the EU and Central Asia in business and research.

Some of these projects, for instance, TELESOL, investigated intelligence-based solutions to support the technology transfer process.

Knowledge Management (KM) has played a critical role in innovation and technology transfer over the past decades. For developing and emerging economies, which are on the way to the knowledge-based society, it is very important to develop knowledge-based decision support systems in different social and business fields.

One of the most popular KM platforms is based on an intelligent agent paradigm. There are a lot of multi-agent based solutions aimed at supporting business applications in different domains. But all these approaches are not related to general concepts of technology transfer and transformation of innovative EU solutions.

Current bottlenecks:

- Modern Information Technology Transfer Models are not related to innovative solutions such as showrooms, webbased frameworks, VR platforms;

- Only a few projects are focused on international cooperation in the ICT area with low and middle income economies, especially, from Central Asia, and they do not provide exhaustive solutions for innovation transformation from the EU research to local markets. 


\section{THE NOVELTY OF THE PROPOSED APPROACH}

The following advance/progress beyond the state of the art that eINTERASIA brings about is identified:

Generic Technology Transfer Concept (TTC) for transformation of the EU research results into innovation of Developing Economies.

The concept is based on the integration of two innovation models - Capability Maturity and Open models. Though the Capability Maturity Model (CMM) comes from the field of software development, it will be used together with the Open Model as a General Model of the maturity in the field of technology transfer. It will help corresponding organizations organize effective communication between the EU and local players in the technology transfer field, formalize and improve the process of knowledge transformation to local needs.

The Concept incorporates the following innovative elements: adapted Capability Maturity Model, ShowRooms, Graph Model and Web-based Framework.

\section{TECHNOLOGY TRANSFER MODEL}

eINTERASIA Transfer Model is based on Capability Maturity Model ISO 15504 and the best current practices in fields such as communication, knowledge management, project management, human resources/organizational development and commercialization of research results.

The Transfer Model helps corresponding organizations characterize their capability in current transfer practice, establish a program of organizational development, and establish a culture of excellence.

Two partner organizations (UniB and MITSOFT) were actively involved in the creation of BONITA transfer model within the Baltic Sea Region INTERREG Program project "Baltic Organization and Network of Innovation Transfer Associations".

eINTERASIA Transfer Model is presented in Fig. 2.

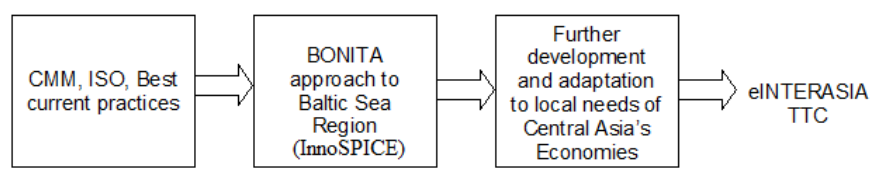

Fig. 2. eINTERASIA Transfer Model.

Three roles act in the field of innovation and technology transfer: technology supplier, technology receiver/acquirer, and technology transfer facilitator (Fig. 3). Organization can perform more than one role in innovation and technology transfer process as well as one role can be performed by more than one organization.

Technology supplier. Technology supplier organizations focus on technology development (in eINTERASIA project RTU, UniB, IFF, MITSOFT and LTR).

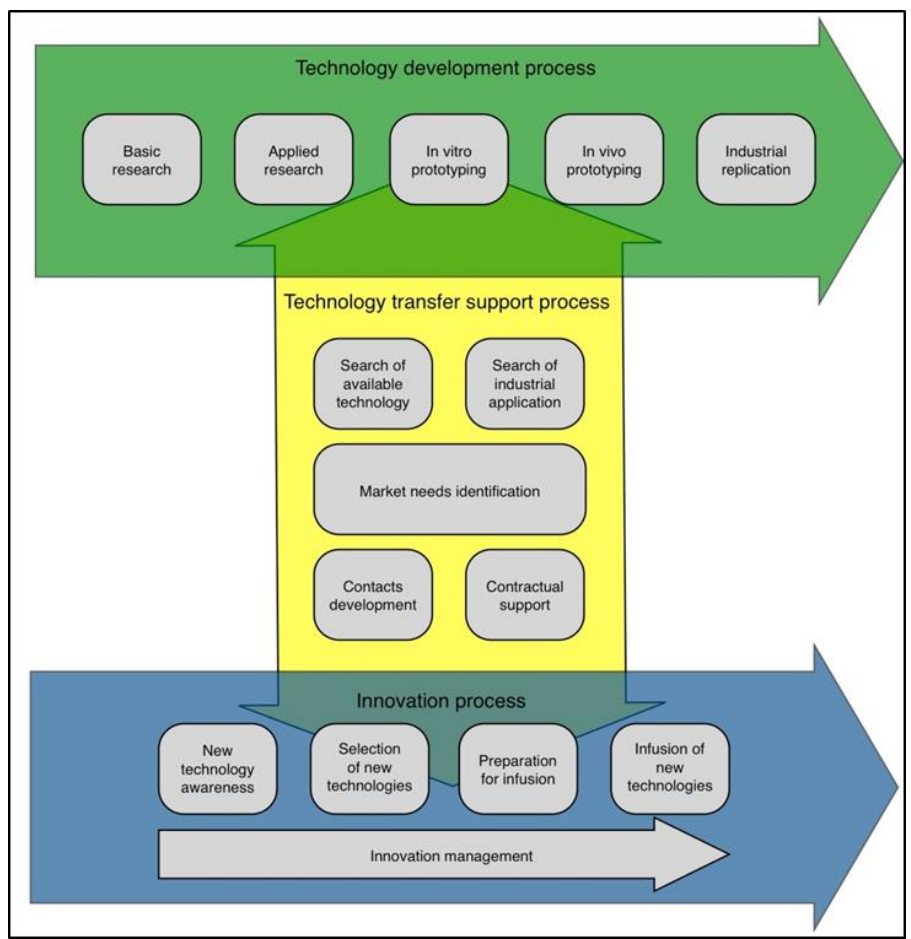

Fig. 3. The roles in technology transfer.

Technology receiver/acquirer. Technology receiver organizations are the ones that take a new technology and implement it to improve their products, services, processes or work environment (in eINTERASIA - UIFA and its members, Astana Innovations, TUT).

Technology transfer facilitator. Technology transfer facilitator organizations are the ones that enable and in many cases drive technology transfer (in our case - TUT and UIFA).

Technology transfer facilitator's activity can range within quite wide limits from a technology transfer broker to a technology transfer driver.

Knowledge commercialization broker's scenario takes place when an active role in knowledge transfer is taken by an intermediary who seeks for knowledge acquirers' needs and knowledge to be transferred and to create a demand-supply data base and/or a network of brokers. The primary action in this activity is to catch the demand. The knowledge supply is secondary. It can be based on the existing knowledge ready for use or can be developed according to the order of acquirer. There are data provided by brokers, whose success ratio is about one of 60 cases. The role of the broker in knowledge commercialization finishes when an agreement for knowledge transfer is concluded between a knowledge acquirer and a supplier. Knowledge commercialization broker's scenario can be classified as low knowledge on the demand side and low knowledge on the supply side. The main risk is up to a knowledge broker that knowledge transfer intermediation efforts will not be successful. The impact of the risk is low because of limited investments into a brokerage activity. 
Knowledge commercialization driver's scenario takes place when full responsibility for knowledge transfer is up to a knowledge transfer driver. The knowledge transfer driver is the one who creates a knowledge commercialization loop with involvement of many players, including knowledge marketing managers, acquirers, developers, sponsors etc. The knowledge transfer driver's activity is based on a knowledge commercialization concept elaborated with the involvement of experts from acquirers, developers, marketing, and funders' communities and on the complementarity of integrated efforts of various capabilities for value creation. Knowledge commercialization driver's scenario can be classified as sufficient knowledge on the demand side and sufficient knowledge on the supply side. The main risk is up to a knowledge transfer driver. The impact of the risk is high because of concentration of responsibility into a driver's activity.

\section{WEB-BASED FRAMEWORK}

A demonstrator of intelligent Web-based Framework (WBF) provides an integrated view on heterogeneous e-resources to support business processes in the selected application domain of transport logistics.

The general structure of web portal is presented in Fig. 4.

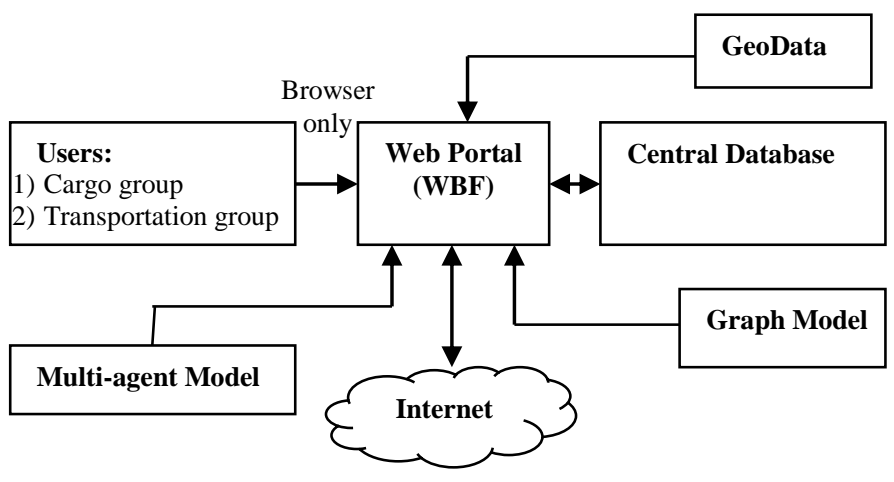

Fig. 4. General structure of web portal.

WBF functioning is based on the following main components:

- www.eLOGMAR.eu prototype;

- Intelligent multi-agent model;

- GeoData approach;

- Graph models and flow programming algorithm.

In comparison with other related projects, a web portal is based on synergy of several innovative components. The proposed approach allows extending a set of portal functions and enhancing the quality of services.

The algorithms of intermodal container transportation are presented in [11].

\section{PROMOTION AND DISSEMINATION}

Two ShowRooms are set up at the premises of partner organizations in Central Asian countries: Tajikistan and Kazakhstan. They serve as marketing and promotional tools to present advances of European IT research results with a double purpose:

- diffusion of research findings and knowledge, making it accessible to people, showing the usability and benefits from solutions in practical applications;

- serving as a forum for experts to attract technology and innovation developers, potential users and beneficiaries, linking researchers, entrepreneurs and policy makers, establishing new strategic initiatives.

The University of Bremen (the Centre for Computing Informatics Technologies), Riga Technical University and MitSoft have already made a good experience with ShowRoom as a part of several regional and European projects. They are used as a prototype in eINTERASIA project.

\section{CONCLUSION}

The brief description of the objectives of International EU project eINTERASIA "ICT Transfer Concept for Adaptation, Dissemination and Local Exploitation of European Research Results in Central Asian Countries" are presented in the paper.

The general structure of the project is broken down into several parts presented by separate papers in this volume of RTU Scientific Journal.

The future research will focus on enhancing IT Transfer Concept and its promotion in different application domains, improvement of the ways of introducing advanced IT solutions in different target markets, including the region of Central Asia.

\section{ACKNOWLEDGEMENT}

The research has been partly supported by the project eINTERASIA "ICT Transfer Concept for Adaptation, Dissemination and Local Exploitation of European Research Results in Central Asian Countries", grant agreement No. 600680 of Seventh Framework Program Theme ICT-9.10.3: International Partnership Building and Support to Dialogues for Specific International Cooperation Actions - CP-SICAINFSO.

\section{REFERENCES}

[1] Lee, D.H. and Richardson, J.J., "A Technology Transfer Model for Industry-University-Government Partnerships," in Proc. of the Engineering Management Conference, 1990. Management through the Year 2000. - Gaining the Competitive Advantage, pp. 352-358, 1990. http://dx.doi.org/10.1109/iemc.1990.201304

[2] Boronowsky, M., Woronowicz, T., Mitasiunas, A., "BONITA - Improve Transfer from Universities for Regional Development," in Proc. of the $3^{\text {rd }}$ ISPIM Innovation Symp., Quebec, Canada, Dec. 12-15, 2010, ISBN 978-951-265-004-7.

[3] Vinitchenko, S., Novickis, L., Smirnov, D., Staniulis, K., Birskis, V., "Demonstration Scenarios of Web Portal with Maritime and Transport Logistics Applications," in Scientific Proc. of the eLOGMAR-M Project. IT \&T Solutions in Logistics and Maritime Applications, Riga, JUMI, pp. 51-62, 2006.

[4] Bluemel, E., Novickis, L., Schumann, M., Leye, S., "Mixed Reality and Digital Engineering solutions and Their Promotion in the Baltic States," 
in 3rd International Workshop Proc. INTEL-EDU, JUMI, Riga, Latvia, Oct. 10, Riga Technical University, pp. 7-19, 2012.

[5] Mitasiunas, A., Ragaisis, S., "Electronic Documents Interoperability Solutions in Academic Environment," in 3rd International Workshop Proceedings INTEL-EDU, Riga, Latvia, Oct. 10, Riga Technical University, pp. 2034, 2012.

[6] Docherty, M., "Primer on open innovation: Principles and practice," PDMA Visions, no. 2, pp. 13-17, 2006.

[7] Preez du N.D., Louw L., "A Framework for Managing the Innovation Process," in Int. Conf. on Management of Engineering \& Technology, pp. 546-558, 2008. http://dx.doi.org/10.1109/PICMET.2008.4599663

[8] Rothwell, R., "Industrial innovation: Success, Strategy, Trends," In M Dodgson and R. Rothwell, (Eds). The Handbook of Industrial Innovation. Aldershot: Edward Elgar, Hants, pp. 33-53, 1995. http://dx.doi.org/10.4337/9781781954201.00010

[9] Trott, P., Innovation Management and New Product Development. 4th ed. Harlow, England: Pearson Education Limited, 2008.

[10] Varjonen, V., "Management of Early Phases in Innovation Process: A Case Study of Commercializing Technology in a Small Enterprise,' M.S. thesis, Helsinki University of Technology, 2006.

[11] Novickis, L., Vinichenko, S., Sotnichoks, M. et al., "Graphs Models and GeoData Based Web portal in Transport Logistics," in The same Scientific Journal of Riga Technical University.
Leonids Novickis is the Head of the Division of Software Engineering. He obtained Dr. sc. ing. degree in 1980 and Dr. habil. sc. ing. degree in 1990 from the Latvian Academy of Sciences. Since 1994, he has been regularly involved in different EU-funded projects: AMCAI (INCO COPERNICUS, 1995-1997) - WP leader; DAMAC-HP (INCO2, 1998-2000), BALTPORTSIT (FP5, 2001-2003), eLOGMAR-M (FP6, 2004-2006) - scientific coordinator; IST4Balt (FP6, 2004-2007), UNITE (FP6, 2006-2008) and BONITA (INTERREG, 2008-2012) - RTU coordinator; LOGIS, LOGISMobile and SocSimNet (Leonardo da Vinci) - partner. He was an independent expert of IST and Research for SMEs in FP6, FP7. He is a corresponding member of the Latvian Academy of Sciences. His research fields include applied software system development, business process modelling, eLogistics, international cooperation, web-based applications. He is the coordinator of FP7 eINTERASIA project.

E-mail: lnovickis@gmail.com

Antanas Mitasiunas is the founder and chairman of the board of company MitSoft. He is also an Associate Professor of the Department of Computer Science at Vilnius University. He obtained a Doctoral Degree in Computer Science (Dr. sc. comp.) from Moscow State University in 1981. He was a national expert of the ICT Committee in FP7, member of advisory board of the international project Enterprice SPICE, expert of national European Structural Funds. Research areas: software design, software quality management, innovations and technology transfer.

E-mail: antanas.mitasiunas@mitsoft.lt 